\title{
Research on the Bilateral Sweep Heuristic for Single Path's Construction
}

\author{
Yao Qifu ${ }^{*}$
}

Department of Mechatronics, Zhejiang Business Technology Institute, Ningbo, Zhejiang, 315012, P.R. China

\begin{abstract}
The team orienteering problem (TOP) is the generation to the case of multi tours of the Orienteering Problem (OP). A pair of origin and destination is specified and a set of potential customers with profits are provided. A single path's initial construction which is considered as the main procedure for feasible solution initialization for the TOP is formulated as an integer program, then the BS heuristic's implementing process is illustrated and the process of bilateral sweep heuristic algorithm (BS) is detailed. Applying the BS to solve the related combinatorial optimization problems appears to be a very promising technique.
\end{abstract}

Keywords: Bilateral sweep heuristic, Single path, Team orienteering problem.

\section{INTRODUCTION}

Collaboration among shippers and carriers for reducing operation costs has become the trend of logistics [1]. Each carrier or freight forwarding entity is also apt to fulfill one's own tasks with others, which means the horizontal collaboration of logistics [2]. Any collaborative carrier does not need to fulfill all his tasks, and may have resource left for the other participating carriers' cooperation. This brings about huge difference from traditional logistic optimization problems, such as the Traveling Salesman Problem (TSP), the Vehicle Routing Problems (VRPs), and etc., which only considers the routing optimization and does not care about the customers' selecting problem. A new situation for all collaborative carriers is that they must determine not only which tasks are to be executed among all the candidates but what the picked tasks' sequence is.

The TOP, which are much closer to practical application than the OP, arises in so many situations, such as the multivehicle version of the home fuel delivery problem, the recruiting of college football players (Butt and Cavalier, 1994), the sports of team orienteering, some applications of pickup or delivery services involving the use of common carriers and private fleets, the service scheduling of routing technicians and multiple days' of personalized mobile tourist guide [3]. The TOP is closely related to vehicle routing problem and Feillet et al. [4] have listed in detail related multi-vehicle routing problems with profits.

Because of the wide application of the TOP, we choose it as a participant carriers' self-optimization model. Remaining tasks are assigned after a member carrier's selection and routing are to be auctioned for other participants' collaboration. As the CTOP is the variant of the TOP considering vehicles' capacity, we only emphasize on the TOP and strive for an effective and efficient heuristic for it.
In the TOP, a fleet of vehicles try to visit a set of customers who are assigned a score. Each vehicle starting from the origin and ending at the destination within a prescribed time (distance) limit tries to visit as many customers. Once a vehicle visits a customer and is awarded the associated score, no other vehicle can be awarded by visiting the same customer [5]. The objective of the TOP is to identify the customers which maximize the fleet of vehicles' collected profit and do not violate each vehicle's given time (distance) limit.

Since the TOP is an NP-hard problem, the research efforts mainly focus on heuristics and meta-heuristics. Butt and Cavalier presented a greedy procedure. Chao et al. [6] proposed a five-step method and a heuristic based on the stochastic algorithm of Tsiligirides. A tabu search algorithm was proposed by Tang and Miller-Hooks. Archetti et al. [7] proposed two tabu search algorithm and a variable neighborhood search algorithm. Vansteenwegen et al. [8] proposed a guided local search meta-heuristic. Until now, only two exact algorithms have been proposed for the TOP: A column generation based algorithm and a branch and price based algorithm, which can only solve problems of very limited size in a reasonable amount of time.

\section{THE FORMULATION OF SINGLE INITIAL PATH'S CONSTRUCTION}

The sweep algorithm has been applied to solve vehicle dispatch problem and VRP. Jacques and Boctor have proposed a sweep-based algorithm for the fleet size and mix vehicle routing problem. To our knowledge, the bilateral sweep heuristic has never been adopted for the TOP though it is inspired by the existing sweep algorithm.

We firstly generate two sweep regions whose polar angles' intervals are

$\left[A^{s p}\left(p^{s d}\right)-A_{\max }, A^{s p}\left(p^{s d}\right)+A_{\max }\right]$

and

$\left[A^{E}\left(p^{s d}\right)-A_{\max }, A^{E}\left(p^{s d}\right)+A_{\max }\right]$ 
determined by the seed vertex's polar angle $A^{s}\left(p^{\text {sd }}\right)$. All vertices dropping into these two regions are defined as the set of $W(W \subset V /\{1, n\})$. Though some vertex belongs into both regions but only one is added into the set $W$. Another corresponding expression for $W$ is the set of the vertices

$$
\begin{aligned}
& (i \in V /\{1, n\}) ; \\
& A^{s p}(i) \in\left[A^{s p}\left(p^{s d}\right)-A_{\max }, A^{s p}\left(p^{s d}\right)+A_{\max },\right. \\
& \left.A^{E p}\left(p^{s d}\right)+A_{\max }\right]
\end{aligned}
$$

Each single path's initial construction which can be considered as a main procedure of feasible solution initialization for the TOP is formulated as an integer program, while an arbitrary vertex $p^{\text {sd }}\left(p^{\text {sd }}=2, \cdots, n-1\right)$ is picked as the seed vertex (seed vertex selection described in Section 4.2) for the path's construction and the corresponding bidirectional sweep region is set as extra constraints.

We associate one binary variable $x_{i j}$ to every edge $(i, j) \in E$, equal to 1 if and only if the corresponding edge is used in the path's construction, and one binary variable $y_{j}$ to every vertex $i \in W$, equal to 1 if and only if the corresponding vertex is in the path. Then each initial path initialization with bidirectional sweep regions' constraints is followed:

$$
\begin{aligned}
& \max \sum_{i \in W} s_{i} y_{i} \\
& \text { s.t. } \sum_{i \in W \cup\{1\}} \sum_{j \in W \cup\{n\}} c_{i j} x_{i j} \leq((i, j) \in E) \\
& \sum_{j \in W \cup\{1\}} x_{i j}=y_{i}(i \in W \cup\{1\}) \\
& \sum_{i \in W \cup\{n\}} x_{i j}=y_{j}(j \in W \cup\{n\}) \\
& \sum_{i, j \in W ; i \neq j} x_{i j} \leq|U|-1(U \subset W \cup\{1, n\} ; 2 \leq|U| \leq|W|) \\
& y_{1}=y_{n}=y_{p^{s d}}=1\left(P^{s d} \in W\right) \\
& x_{i j} \in\{0,1\}((i, j) \in E ; i \in W \cup\{1\} ; j \in W \cup\{n\}) \\
& y_{i} \in\{0,1\}(i \in W)
\end{aligned}
$$

The objective function (1) maximizes the collected profit from visiting customer vertices. Constraint (2) describes each path's time (distance) restriction. Constraints (3) and (4) are the so-called assignment constraints. Constraint (5) eliminates any sub tours that do not involve both depots. Constraint (6) ensures origin depot, destination depot and the seed customer vertex are all in the initial path. Constraints (3), (4) and (6) jointly ensure each path starts from vertex 1 and ends at vertex $n$ passing through the seed vertex.
Constraints (7) and (8) set the integral requirement on each variable.

\section{THE BS HEURISTIC FOR SINGLE PATH'S CONSTRUCTION}

In the class of combinatorial problems, such as VRP, OP, TOP and etc. each path (tour) must start from the origin and end at the destination. The shape of each path (tour) is mainly influenced by the farthest selected customer vertex, which determines not only the part from the origin to itself but the other part from itself back to the destination. Simple or random construction strategy often induces the path to an uncontrolled direction, which may cause inefficiency of next improvement strategy.

The bilateral sweep heuristic applies the strategy based on a seed vertex which determines the path's rough direction. Once a seed vertex for path's initial construction is picked, the bidirectional sweep and insertion are implemented simultaneously according to the partial path from origin to the seed customer vertex and the partial path from the seed vertex back to the destination. An example is given (Fig. 1).

We illustrate the BS heuristic's implementation process in (Fig. 1) step by step. In (Fig. 1a), an initial path is constructed as S-5-E, which is determined by the seed vertex 5; In (Fig. 1b), vertex 7 is inserted into the path when only one qualified vertex was swept, and the path becomes S-7-5-E; In (Fig. 1c), vertex 8 which acts as a more efficient vertex is inserted into the path when candidate vertex 4 and 6 are both qualified, then the path is S-7-5-8-E; In Fig. (1d), vertex 4, which is a better one, is inserted into the path through vertex 6 is swept again, and the path turns to be S-7-5-4-8-E. In practice, the BS heuristic usually implements more iterations than exhibited 3 times.

The BS is processed in five steps which are detailed as followed.

Initialization: determine initial path's seed vertex and related procedures' parameters.

(1) Select a seed customer vertex $p^{\text {sd }}$.

(2) Choose a stopping condition, max number of iteration $B_{\text {max }}=\left[A_{\max } / 2 a_{\text {step }}\right]$.

(3) Set each sweep step $a_{\text {step }}$ and $k \leftarrow 0$.

Bilateral Sweep: repeat the following sequence

(1) Set $k \leftarrow k+1$;

(2) If $k \leq B_{\max }$

Step 1 (Candidate). Record all candidate vertices located in the bidirectional regions which fall into

$\left[A^{s p}\left(p^{s d}\right)-k \cdot a_{\text {step }}, A^{s p}\left(p^{s d}\right)+k \cdot a_{\text {step }}\right]$

Or 
$\mathbf{a}$
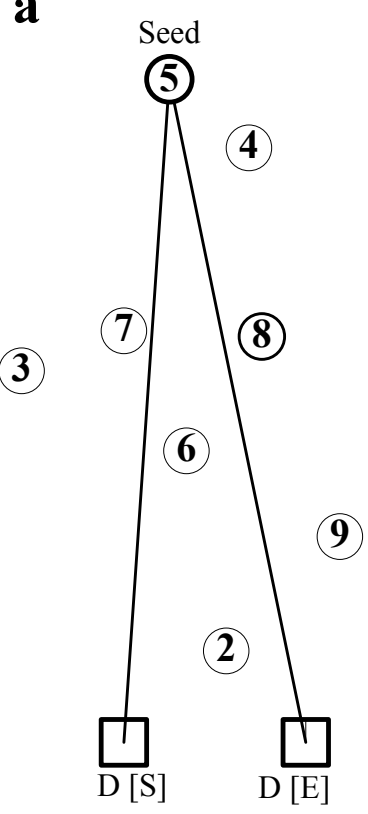

c

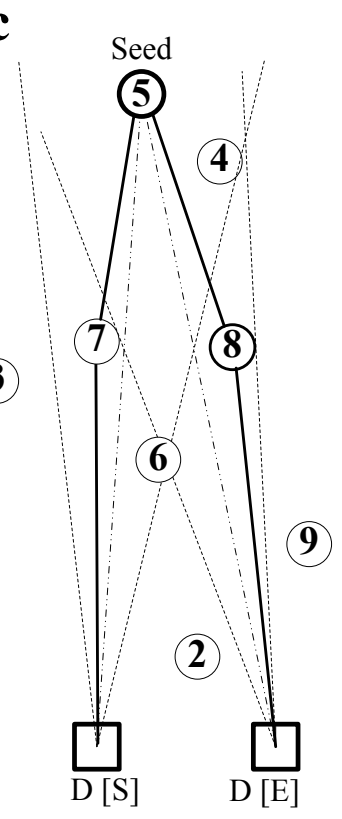

b

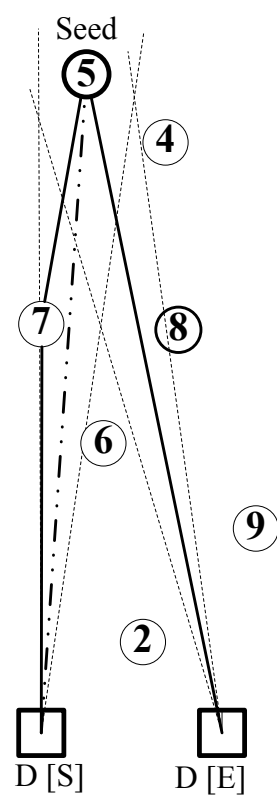

9

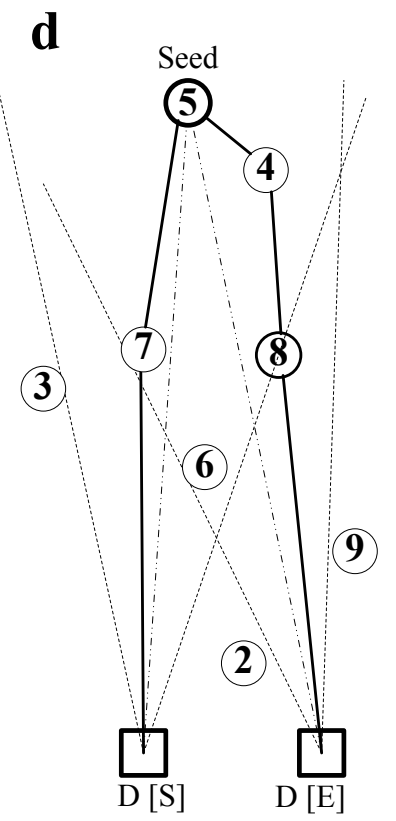

Fig. (1). An illustration of the BS's implementing process in an order of (a) to (d).

$$
\left[A^{E p}\left(p^{s d}\right)-k \cdot a_{\text {step }}, A^{E p}\left(p^{s d}\right)+k \cdot a_{\text {step }}\right]
$$

Step 2 (Locate). Find swept vertices' best location in the initial path where minimal increasing time (distance) is generated;

Step 3 (Qualify). Select all the vertices which do not violate the given time (distance) limit after inserting them in corresponding initial path;

Step 4 (Evaluate). Choose the most efficient vertex which initiates the minimal additional time (distance) and are within the time (distance) limit;
Step 5 (Execute). Insert the chosen vertex into its best location, if it exists, and renew all other swept vertices to the remaining set of candidate customer vertices.

\section{CONCLUSION}

As sweep algorithm, bilateral sweep heuristic is also based on the vertices' polar angle and radius. Because the origin depot and the destination depot are usually not identical, two polar coordinate systems are set up to locate all vertices. One polar coordinate system chooses origin depot as the origin, and we define it as the Start polar coordinate 
system. The other one chooses the destination depot as the origin, and we define it as the End polar coordinate system. Both polar coordinate systems define the axis which crosses both the origin depot and the destination depot as original polar angle (angle 0), so vertices' polar angles and radii in two polar coordinate systems are different. By researching the proposed bilateral sweep heuristic algorithm, the implementing process is illustrated. Applying the BS to solve other related combinatorial optimization problems appears to be a very promising method.

\section{ABOUT THE AUTHOR}

Yao Qifu is a professor in Zhejiang Business Technology Institute. The author's major is Computer science and Engineering.

\section{CONFLICT OF INTEREST}

The author confirms that this article content has no conflict of interest.

\section{ACKNOWLEDGEMENTS}

This work was financially supported by the Ningbo Natural Science Funds (2013A610065).

\section{REFERENCES}

[1] E.O. Kuyzu and G.M. Savelsbergh, "Shipper collaboration", Comput. Oper. Res., vol. 6, pp. 1551-1560, 2007.

[2] M.A. Krajewska, and H. Kopfer,"Collaborating freight forwarding enterprises”, OR Spect., vol. 28, pp. 301-317, 2006.

[3] P. Vansteenwegen, W. Souffriau, G.V. Berghe and D.V Oudheusden, "A guided local search metaheuristic for the team orienteering problem", Eur. J. Operat. Res., vol. 196, pp. 118-127, 2009.

[4] H. Tang and E. Miller-Hooks, "A tabu search heuristics for the team orienteering problem", Comput. Operat. Res., vol. 32, pp. 1379-1407, 2005.

[5] D. Feillet, P. Dejax and M. Gendreau, "Traveling salesman problems with profits", Transport. Sci., vol. 39, pp. 188-205, 2005.

[6] I. Chao, B. Golden and E. Wasil, "The team orienteering problem", Eur. J. Operat. Res., vol. 88, pp. 464-474, 1996.

[7] C. Archetti, D. Feillet, A. Hertz and M.G. Speranza, "The capacitated team orienteering and profitable tour problems", J. Oper. Res. Soc. vol. 60, pp. 831-842, 2009.

[8] P. Vansteenwegen, W. Souffriau, G.V. Berghe and D.V. Oudheusden, "A guided local search metaheuristic for the team orienteering problem", Eur. J. Operat. Res., vol. 196, pp. 118-127, 2009.

Received: September 22, 2014

Revised: November 03, 2014

Accepted: November 06, 2014

(C) Yao Qifu; Licensee Bentham Open.

This is an open access article licensed under the terms of the Creative Commons Attribution Non-Commercial License (http://creativecommons.org/licenses/by-nc/3.0/) which permits unrestricted, non-commercial use, distribution and reproduction in any medium, provided the work is properly cited. 